\title{
Describing acceptable objects by means of Sugeno integrals
}

\author{
Henri Prade \\ Agnès Rico \\ Institut de Recherche en Informatique de Toulouse (IRIT) Équipe de Recherche en Ingénierie des Connaissances (ERIC) \\ Université Paul Sabatier \\ Toulouse, France \\ Université Claude Bernard Lyon 1 \\ prade@irit.fr \\ Lyon, France \\ agnes.rico@univ-lyon1.fr
}

\begin{abstract}
Objects are usually described by combinations of properties. Logic-based descriptions offer compact representations for binary properties. Besides, Sugeno integrals are wellknown as a powerful qualitative aggregation tool in multiplecriteria decision, which is applicable to gradual properties, and takes into account positive synergies between properties. The paper proposes to investigate the potential use of Sugeno integrals as a representation tool, to lay bare their relation with possibilistic logic representations, and to discuss the handling of negative synergies in this setting using a pair of Sugeno integrals.
\end{abstract}

Keywords-Sugeno integral; possibilistic logic; formal concept analysis; pattern structures.

\section{INTRODUCTION}

The description of objects has been addressed from different points of view. In formal concept analysis [10], the association between objects and properties is given under the form of a simple relation, without any background knowledge about possible logical links between properties. Rather, the usually complete information about the properties possessed by the considered objects is the basis for defining formal concepts as pairs of sets of properties associated bi-univoquely with sets of objects, and then discovering association rules between concepts (which hold for the objects).

In formal concept analysis, we are dealing with factual knowledge about objects (or class of objects). This knowledge just corresponds to the conjunction of properties satisfied by each object. However, this knowledge may become incomplete (e.g., it is just known that object $x$ satisfies $p \vee p^{\prime}$ ), when we go from basic formal concept analysis to pattern structures [8], [9].

Describing objects may serve other purposes. Rather than reporting what is known about available objects, one may describe objects that we are looking for (without even knowing it they exist). In the first case, we are representing knowledge, while in the second case we are specifying preferences. In each case, we are not interested in exactly the same problems. When dealing with knowledge, one may try to conceptualize, classify, or reason, while in the case of preferences, we are usually facing some decision problem.
Sugeno integrals [16], [17] were originally proposed as a family of sophisticated (and powerful) qualitative aggregation operators in multiple criteria decision. They apply to gradual properties graded in the unit interval $[0,1]$, and they can capture positive synergies between properties by means of weights that can be allocated to any subset of properties (not only to single properties). Thus, one can express that satisfying several properties together is strictly better that what is conveyed by the satisfaction degrees associated to the individual properties (and their weights). Moreover, since the unit interval $[0,1]$ can be viewed as a totally ordered lattice with operations $\wedge$ and $\vee$, it has been noticed [15], [3] that any lattice polynomial $P\left(x_{1}, \ldots, x_{n}\right)$ with variables $x_{1}, \ldots, x_{n}$ (representing the degrees of fulfillment of $n$ properties), involving any constants $c_{1}, \ldots, c_{m}$ in $[0,1]$, and formed in the usual manner with $\wedge, \vee$, and parentheses, is a Sugeno integral provided that the limit conditions $P(1, \ldots, 1)=1$ and $P(0, \ldots, 0)=0$ hold. Thus, Sugeno integrals include as particular cases Boolean combination of properties, involving no negation. This suggests that Sugeno integrals may be viewed not only as an evaluation tool, but also as a representation tool for either describing preferences or knowledge.

This may be paralleled with a possibilistic logic [6] description where any logical combination of propositions can be weighted (each positive literal may be thought as representing a property), which enables us to express both positive and negative synergies between literals. But, the extension of possibilistic logic to gradual properties, even if proposals exist, is not straightforward.

These remarks suggest to further investigate Sugeno integrals as a representation tool for describing more or less acceptable (or satisfactory) objects, in relation with compact logical representations. The paper is organised as follows. Section 2 starts with the introduction of a basic possibility assignment assessing how acceptable is any object completely described in terms of a set of properties. In case of binary properties, this is nothing but a possibility distribution over a set of interpretations underlying a possibilistic logic base. This basic possibility assignment gives birth to a fuzzy measure, from which a Sugeno integral can be defined. Section 3 shows how the Sugeno integrals reflect 
the description of the objects. Some illustrative examples are provided in three situations of interest: i) when the properties and the basic possibility assignment are binary; ii) when the properties are binary and the basic possibility assignment is graded, and iii) when both are graded. Section 4 proposes a way for accommodating negative synergies, which cannot be captured by a Sugeno integral (while they can be captured in possibilistic logic for binary properties), using a pair of Sugeno integrals. Section 5 discusses questions and lines for further research.

\section{DESCRIBING OBJECTS BY THEIR PROPERTIES}

One considers a set of properties $\mathcal{P}=\left\{p_{1}, \ldots, p_{n}\right\}$, considered as relevant for describing a set of objects $\mathcal{X}$. It is assumed that $\mathcal{P}$ contains all the necessary properties for describing the objects under consideration. An object is denoted by $x$. Each object $x$ is evaluated according to each property. So $x$ can be viewed as a vector $\left(x_{1}, \ldots, x_{n}\right)$ where $x_{i} \in[0,1]$ is the degree to which $x$ satisfies property $p_{i}$. In what will be referred to in the following as the binary case, the property degrees take only the values 1 or 0 : each property is true or false for $x$.

In this paper we are working with what may be called a basic possibility assignment [4], i.e. a mapping $\sigma: 2^{\mathcal{P}} \rightarrow$ $[0,1]$. We first consider the binary case where properties are true or false. In this case, $\sigma$ estimates the extent $\sigma(T)$ to which one can find acceptable an object that satisfies exactly all the properties in subset $T$, and no others (note that in this assignment, a set should be understood conjunctively). In such a view, $\sigma(\{p\})$, for instance, estimates to what extent an object satisfying $p$ only is acceptable. It might be viewed as a kind of "degree of importance" of property $p$ alone. Thus, let $T$ be a subset of properties $\left\{p_{(1)}, \cdots, p_{(k)}\right\} \subseteq \mathcal{P}$, then $\sigma(T)=1$ means that if an object $x$ satisfies properties $p_{(1)}, \cdots$, and $p_{(k)}$, which may be denoted $p_{1}(x) \wedge \cdots \wedge p_{k}(x)$, $x$ is (fully) acceptable. Conversely, $x$ is fully acceptable if $\exists S$ s.t. $\sigma(S)=1$ and $x$ satisfies all properties in $S$ and no others. More generally $x$ may be acceptable at an intermediary level $0<\sigma(T)<1$. Clearly there may be several distinct $T_{i}, T_{j}$ such that $\sigma\left(T_{i}\right)>0, \sigma\left(T_{j}\right)>0$. We assume the normalization condition $\bigvee_{T \subset \mathcal{P}} \sigma(T)=1$. This condition implies that there exists a set $\bar{T}$ of properties satisfying $\sigma(T)=1$. This means that there exists at least one configuration of properties that is considered as fully satisfactory or acceptable.

Note that one may represent different situations with respect to two subsets $T$ and $T^{\prime}$ such that $T \cap T^{\prime}=\emptyset$. Namely, one may have

- $\sigma(T)=\sigma\left(T \cup T^{\prime}\right)$, which means that having the properties in $T^{\prime}$ satisfied or not, when those in $T$ are satisfied does not influence the level of acceptability;

- $\sigma(T)<\sigma\left(T \cup T^{\prime}\right)$, which expresses a positive influence of $T^{\prime}$ on $T$ : satisfying all the properties in $T \cup T^{\prime}$ (and no others) is better than satisfying only the ones in $T$;
- $\sigma\left(T \cup T^{\prime}\right)<\sigma(T)$, which expresses a negative influence of $T^{\prime}$ on $T$ : satisfying all the properties in $T \cup T^{\prime}$ (and no others) is worst than satisfying only the ones in $T$.

When $\max \left(\sigma(T), \sigma\left(T^{\prime}\right)\right)<\sigma\left(T \cup T^{\prime}\right)$, there is a positive synergy between $T$ and $T^{\prime}$, while $\min \left(\sigma(T), \sigma\left(T^{\prime}\right)\right)>$ $\sigma\left(T \cup T^{\prime}\right)$ reveals a negative synergy.

It is worth noticing that any subset $T \subseteq \mathcal{P}$ is nothing but an interpretation from a logical point of view, with respect to the language induced by the properties in $\mathcal{P}$ viewed as propositional literals. Indeed $T$ represents the state where all properties in $T$ are true, and all properties in $\mathcal{P}-T$ are false. Thus, an object is described by the set of properties it possesses (the properties that it misses do not need to be listed: we know them by complementation).

Thus, $\sigma$ is just a possibility distribution over a set of interpretations (note that e.g., $\{p, q\}$ is just another notation of the interpretation $p q \neg r$ if $\mathcal{P}=\{p, q, r\}$ ). Any such normalized distribution can be viewed as the semantic counterpart of a consistent possibilistic logic base [6].

Example 1 Assume we have 3 properties: $\mathcal{P}=\{p, q, r\}$. We want to state that acceptable objects are either those which have properties $p$ and $q$, or to a less extent those with properties $p$ and $r$ and nothing else. Thus acceptable objects are described by: $\sigma(\{p, q\})=1, \sigma(\{p, r\})=\alpha<1$ and $\sigma(S)=0$ for any other subset $S$ of $\mathcal{P}$. $\sigma$ is semantically associated with the possibilistic logic base ${ }^{1} \mathcal{A}^{N}$ describing the acceptable objects more compactly ${ }^{2}$ :

${ }^{1}$ Let $B=\left\{\left(\varphi_{i}, \alpha_{i}\right) \mid i=1, n\right\}$ be a propositional possibilistic logic base, where $\varphi_{i}$ denotes a classical logical formula and its weight $\alpha_{i} \in[0,1]$. Each formula $\left(\varphi_{i}, \alpha_{i}\right)$ should be understood as $N\left(\varphi_{i}\right) \geq \alpha_{i}$, where $N$ is a necessity measure obeying the characteristic property $N(\varphi \wedge \psi)=$ $\min (N(\varphi), N(\psi))$. A necessity measure is associated with a possibility distribution $\pi$ by the formula $N(\varphi)=\min _{\omega \notin M(\varphi)} 1-\pi(\omega)=1-$ $\Pi(\neg \varphi)$, where $\Pi$ denotes the dual (potential) possibility measure, and $M(\varphi)$ is the set of interpretations for which $\varphi$ is true. The possibilistic logic base $B$ is semantically associated with the possibility distribution

$$
\pi_{B}(\omega)=\min _{i=1, n} \pi_{\left(\varphi_{i}, \alpha_{i}\right)}(\omega)
$$

with $\pi_{\left(\varphi_{i}, \alpha_{i}\right)}(\omega)=1$ if $\omega \in M\left(\varphi_{i}\right)$, and $\pi_{\left(\varphi_{i}, \alpha_{i}\right)}(\omega)=1-\alpha_{i}$ if $\omega \notin$ $M\left(\varphi_{i}\right)$. Here an interpretation $\omega$ is all the more possible as it does not violate any formula $p_{i}$ with a high priority level $\alpha_{i}$.

${ }^{2} \mathrm{~A}$ slightly more compact representation in this case is given by $\mathcal{A}^{\Delta}=$ $\{[p \wedge q \wedge \neg r, 1],[p \wedge \neg q \wedge r, \alpha]\}$, where the information is now represented by formulas denoted $[\psi, \gamma]$, which expresses the constraint $\Delta(\psi) \geq \gamma$, where $\Delta$ denotes a measure of actual possibility defined from a possibility distribution $\pi$ by $\Delta(\psi)=\min _{\omega \in M(\psi)} \pi(\omega)$. As a consequence, th measures of actual possibility obey the following characteristic postulate: $\Delta(\varphi \vee \psi)=\min (\Delta(\varphi), \Delta(\psi))$. Consequently, $\Delta$ is decreasing with respect to logical entailment, which contrasts with necessity (or potential possibility) measures. In other words, the piece of information $[\psi, \gamma]$ expresses that any model of $\psi$ is at least possible with degree $\gamma$. Conversely, let $P=\left\{\left[\psi_{j}, \gamma_{j}\right] \mid j=1, k\right\}$ be a $\Delta$-possibilistic logic base where $\left[\psi_{j}, \gamma_{j}\right]$ is understood as $\Delta\left(\psi_{j}\right) \geq \gamma_{j}$. Its semantics is given by the possibility distribution

$$
\pi_{P}(\omega)=\max _{j=1, k} \pi_{\left[\psi_{j}, \gamma_{j}\right]}(\omega)
$$

with $\pi_{\left[\psi_{j}, \gamma_{j}\right]}(\omega)=0$ if $\omega \notin M\left(\psi_{j}\right)$, and $\pi_{\left[\psi_{j}, \gamma_{j}\right]}(\omega)=\gamma_{j}$ if $\omega \in M\left(\psi_{j}\right)$.

Note that $\pi_{P}$ is obtained as the max-based disjunctive combination of the representations of each formula in $P$, while $\pi_{B}$ is obtained by a min-based conjunctive combination of the representations of each formula in $B$. 


$$
\mathcal{A}^{N}=\{(p, 1),(q \vee r, 1),(q, 1-\alpha),(\neg q \vee \neg r, 1)\} .
$$

This is an example of negative synergy between $q$ and $r$. Suppose now that acceptable objects are either those which have properties $p$ and $q$ (and possibly $r$ ), or to a less extent those with properties $p$ and $r$, then now $\sigma^{\prime}(\{p, q\})=$ $\sigma^{\prime}(\{p, q, r\})=1, \sigma^{\prime}(\{p, r\})=\alpha<1$ and $\sigma^{\prime}(S)=0$ for any other subset $S$ of $\mathcal{P}$. This corresponds to the possibilistic logic base $\mathcal{A}^{\prime N}=\{(p, 1),(q \vee r, 1),(q, 1-\alpha)\}$. Lastly, $\sigma^{\prime \prime}(\{p, q, r\})=1, \sigma^{\prime \prime}(\{p, q\})=\sigma^{\prime \prime}(\{p, r\})=\alpha<1$ and $\sigma^{\prime \prime}(S)=0$ for any other subset $S$, corresponding to $\mathcal{A}^{\prime \prime N}=\{(p, 1),(q \vee r, 1),(q, 1-\alpha),(r, 1-\alpha)\}$ is a case of positive synergy between $q$ and $r$ in context $p$.

Suppose now that the property degrees belong to $[0,1]$. Then $\sigma$ estimates the extent $\sigma(T)$ to which the subset $T$ of (gradual) properties is important as a whole for the acceptability of an object.

On the power set of $\mathcal{P}$, a basic possibility assignment $\sigma$ induces the following fuzzy measure (i.e., a set function increasing in the broad sense with inclusion)

$$
\mu(T)=\max _{S \subseteq T} \sigma(S) .
$$

In such a context, $\mu(T)=1$ means that $T$ contains an important subset of properties, i.e. $\exists S \subseteq T$ such that $\sigma(S)=1$. In the following, $\mu(T)$, (resp. $\sigma(T)$ ) will be simply noted $\mu_{T}$ (resp. $\sigma_{T}$ ).

\section{SugENO INTEGRALS}

In multiple criteria decision, a Sugeno integral [12] is generally used for evaluating to what extent an object, which is precisely described by the degrees to which it satisfies the different properties, is globally acceptable. A Sugeno integral is a monotonically increasing aggregation function: it is all the greater as properties are more satisfied. Thus, although it can encode any Boolean combination of conjunction and disjunction operators (without negations anywhere), as already said, it is not surprising that it remains less expressive than a possibilistic logic base, since it cannot account for negative influence and synergy.

\section{A. Definitions and properties}

Let $x=\left(x_{1}, \ldots, x_{n}\right) \in[0,1]^{n}$ be an object and $\mu$ be a fuzzy measure on the power set. The Sugeno integral of $x$ with respect to $\mu$ is:

$$
\oint_{\mu}(x)=\bigvee_{T \subseteq \mathcal{P}}\left[\mu_{T} \wedge\left(\bigwedge_{i \in T} x_{i}\right)\right]=\bigwedge_{T \subseteq \mathcal{P}}\left[\mu_{\mathcal{P} \backslash T} \vee\left(\bigvee_{i \in T} x_{i}\right)\right]
$$

The two expressions of a Sugeno integral may be viewed as counterparts of the actual possibility-based and the necessity-based readings of a possibility distribution respectively (see footnotes 1 and 2).

Let $\sigma$ be a basic possibility assignment and $\mu$ be the associated fuzzy measure. We assume that $\sigma(\emptyset)=0$ (which agrees with the increasing nature of Sugeno integrals: an object is all the more satisfactory as it satisfies all the properties at a high degree). Indeed a Sugeno integral cannot express that acceptable objects are the ones that do not satisfy any property. We further assume that $\vee_{T \subseteq \mathcal{P}} \sigma(T)=1$ (which is the normalization condition). Then we have

Proposition 1 If $\sigma(\emptyset)=0$ and $\vee_{T \subseteq \mathcal{P}} \sigma(T)=1$ then

$$
\oint_{\mu}(x)=\bigvee_{T \subseteq \mathcal{P}}\left(\sigma_{T} \wedge\left(\bigwedge_{i \in T} x_{i}\right)\right)
$$

Proof According to [15], there exists a set function $c: 2^{\mathcal{P}} \rightarrow[0,1]$ such that $\oint_{\mu}=W_{c}^{\vee \wedge}$ on $[0,1]$ where $W_{c}^{\vee \wedge}$ is a weighted max-min function defined by: $W_{c}^{\vee \wedge}(x)=$ $\bigvee_{T \subset \mathcal{P}}\left[c_{T} \wedge\left(\bigwedge_{i \in T} x_{i}\right)\right]$

$\bar{W}_{\sigma}^{\vee \wedge}$ and $\oint_{\mu}$ are equal on the characteristic functions which is equivalent to $W_{\sigma}^{\vee \wedge}=W_{c}^{\vee \wedge}$ are equal on the characteristic functions. According to [15], this property is equivalent to $W_{\sigma}^{\vee \wedge}=W_{c}^{\vee \wedge}$ on $[0,1]^{n}$. So $\oint_{\mu}(x)=W_{\sigma}^{\vee \wedge}(x)$ fo all $x$ in $[0,1]^{n}$.

Equation (1) entails the following consequences.

- $\oint_{\mu}(x)=1$ if and only if $x$ satisfies a set of properties $T$ such that $\sigma(T)=1$. The biggest score given by the Sugeno integral is equivalent to fully satisfy all the properties of a maximally important subset.

- $\oint_{\mu}(x)=0$ if and only if $\forall T$ such that $\sigma(T) \neq 0 \exists i$ such that $x_{i}=0$. In particular, $\forall T$ such that $\sigma(T)=1$ $\exists i$ such that $x_{i}=0$. The smallest score obtained by the Sugeno integral implies that $x$ doesn't satisfy at least one property in any important subset.

- $\oint_{\mu}(x)=\alpha$ with $\left.\alpha \in\right] 0,1[$ if and only if there exists a set of properties $T$ such that $\sigma(T) \wedge\left(\wedge_{i \in T} x_{i}\right)=\alpha$. So $x$ satisfies at least at level $\alpha$ a property belonging to a set having at least an importance $\alpha$. In particular, for sets such that $\sigma(T)=1$, we have $\wedge_{i} x_{i} \leq \alpha$. When the score given by the Sugeno integral equals $\alpha$, it entails that there is at least one property in each maximally important subset that is not satisfied by $x$ over level $\alpha$.

If acceptable objects are supposed to have some properties, this can be denoted with a disjunction of conjunctions of properties: $x$ has to satisfy $\left(p_{h} \wedge \ldots \wedge p_{i}\right) \vee\left(p_{j} \wedge \ldots \wedge p_{k}\right) \vee$ $\ldots\left(p_{l} \wedge \ldots \wedge p_{m}\right)$. Note that a property may appear in many conjunctions. We consider all conjunctions of properties as important subsets, i.e. we choose $\sigma\left(\left\{p_{h}, \ldots, p_{i}\right\}\right)=$ $\sigma\left(\left\{p_{j}, \ldots, p_{k}\right\}\right)=\ldots \sigma\left(\left\{p_{l}, \ldots, p_{m}\right\}\right)=1$ and $\sigma$ is equal to 0 otherwise. Hence acceptable objects satisfy $\oint_{\mu}(x)=1$ where $\mu$ is the fuzzy measure associated to $\sigma$.

If acceptable objects are supposed to not have some properties, this can be similarly modeled. Then acceptable objects satisfy $\oint_{\mu}(x)=0$ where $\mu$ is the fuzzy measure associated to the $\sigma$ which describes these properties.

To illustrate these points, let us consider 3 cases of increasing complexity:

1) properties are binary, $\sigma$ takes its values in $\{0,1\}$,

2) properties are binary, $\sigma$ takes its values in $[0,1]$,

$3)$ properties are gradual, $\sigma$ takes its values in $[0,1]$. 


\section{B. Illustration}

1) Binary properties with $\{0,1\}$-valued measures: Example 2.1 $\mathcal{P}=\left\{p_{1}, p_{2}, p_{3}, p_{4}\right\}$ and acceptable objects are supposed to have property $p_{2}$ only. Thus $\sigma\left(\left\{p_{2}\right\}\right)=1$ and 0 otherwise. In such a case

$$
\begin{aligned}
& \oint_{\mu}\left(x^{1}\right)=\oint_{\mu}(1,1,1,1)=1, \\
& \oint_{\mu}\left(x^{2}\right)=\oint_{\mu}(1,0,1,0)=0, \\
& \oint_{\mu}\left(x^{3}\right)=\oint_{\mu}(1,1,0,0)=1, \\
& \oint_{\mu}\left(x^{4}\right)=\oint_{\mu}(0,1,1,1)=1 .
\end{aligned}
$$

Objects $x^{1}, x^{3}$ and $x^{4}$ are accepted by $\oint_{\mu}$, which implicitly embeds positive synergy.

Note that if acceptable objects are supposed to not have property $p_{2} x_{2}$ would be the only accepted object.

2) Binary properties with $[0,1]$-valued measures:

Example 2.2 We keep the same properties and the same objects as in the previous example. Acceptable objects are now supposed to have properties $p_{1}$ and $p_{2}$ with a bigger importance for satisfying $p_{1}$. So we take $\sigma\left(\left\{p_{1}\right\}\right)=1$ $\sigma\left(\left\{p_{2}\right\}\right)=0.5$ and 0 otherwise. In such a case we have

$\oint_{\mu}\left(x^{1}\right)=\oint_{\mu}(1,1,1,1)=1$,

$\oint_{\mu}\left(x^{2}\right)=\oint_{\mu}(1,0,1,0)=1$,

$\oint_{\mu}\left(x^{3}\right)=\oint_{\mu}(1,1,0,0)=1$,

$\oint_{\mu}\left(x^{4}\right)=\oint_{\mu}(0,1,1,1)=0.5$.

So $x^{1}, x^{2}$ and $x^{3}$ are accepted (by $\oint_{\mu}$ ).

Note that if acceptable objects are supposed to not have property $p_{1}$ or $p_{2}$, no object would be accepted.

3) Gradual properties with [0,1]-valued measures:

Example 2.3 We keep the same properties as in the previous examples. Acceptable objects are again supposed to have properties $p_{1}$ and $p_{2}$ with a bigger preference for satisfying $p_{1}$. The difference is that the objects are evaluated in $[0,1]$. So we take $\sigma\left(\left\{p_{1}\right\}\right)=1 \sigma\left(\left\{p_{2}\right\}\right)=0.5$ and 0 otherwise. In such a case we have

$$
\begin{aligned}
& \oint_{\mu}\left(x^{1}\right)=\oint_{\mu}(0.5,1,1,1)=0.5, \\
& \oint_{\mu}\left(x^{2}\right)=\oint_{\mu}(1,0.5,1,0)=1, \\
& \oint_{\mu}\left(x^{3}\right)=\oint_{\mu}(1,1,0,0)=1, \\
& \oint_{\mu}\left(x^{4}\right)=\oint_{\mu}(0,1,0.4,0.2)=0.5 . \\
& \text { So } \left.x^{2} \text { and } x^{3} \text { are accepted (by } \oint_{\mu}\right) .
\end{aligned}
$$

Note that if acceptable objects are supposed to not have property $p_{1}$ or $p_{2}$, no object would be accepted. But we can now be soft. Note that $x^{1}$ and $x^{4}$ are acceptable to a less extent, since property $p_{2}$ is satisfied, but $p_{2}$ is less important than $p_{1}$, and the most important property is satisfied only at the level 0.5 by $x^{1}$, or even not satisfied for $x^{4}$.

\section{ACCOMMODATING NEGATIVE SYNERGIES}

We now discuss the problem of the representation of negative synergies, and then propose a way for handling them.

\section{A. Presentation of the problem}

As shown in the previous section, acceptable objects are supposed to have some subsets of properties, and any object satisfying a set of properties containing one of these subsets will be at least as much accepted from a Sugeno integral representation point of view.

Let us consider an example already used in Section II.

Assume we have 3 properties $\mathcal{P}=\{p, q, r\}$. We want to state that acceptable objects are either those which have properties $p$ and $q$, or properties $p$ and $r$ and nothing else. Thus, in this example acceptable objects are described by $\{p, q\}$ or by $\{p, r\}$. This gives birth to a possibility distribution on $2^{\mathcal{P}}: \sigma(\{p, q\})=\sigma(\{p, r\})=1$ and $\sigma(S)=0$ for any other subset $S$ of $\mathcal{P}$, associated with the propositional logic base $\mathcal{A}$ describing the acceptable objects:

$\mathcal{A}=\{p, q \vee r, \neg q \vee \neg r\}$

Then, let $\mu$ be the fuzzy measure on $\mathcal{P}$ associated with $\sigma$. Let $x=(1,1,1)$ be an object having properties $p, q, r$ we have $\oint_{\mu}(x)=1$.

Thus, the Sugeno integral does not acknowledge the fact that such an object is not satisfactory. Indeed satisfying $p$, $q$, and $r$ cannot be worse than satisfying $p$, and $q$, from a Sugeno integral point of view.

Generally speaking, if a Boolean expression made of conjunctions and disjunctions also involve negative literals, one may replace them by renaming them, e.g., $(p \wedge q) \vee \neg p$ can be rewritten $q \vee \neg p$, and finally $q \vee p^{\prime}$, where $p^{\prime}$ denotes $\neg p$. But, there are Boolean expressions where a literal appears both positively and negatively whatever the form the expression is written. It is the case for instance for $(p \wedge q) \vee(\neg p \wedge \neg q)$ which is still equivalent to $(p \vee \neg q) \wedge(\neg p \vee q)$. In such a case it is possible to replace the latter expression by $\left(p \vee q^{\prime}\right) \wedge\left(p^{\prime} \vee q\right)$, but this increases the number of properties, and we would have to separately maintain that no object can be both $p$ and $p^{\prime}$, or $q$ and $q^{\prime}$. In such a case, a Sugeno integral may acknowledge the fact that an object which satisfies $p$ and $q$ is less acceptable than one which satisfies $p$ and $q^{\prime}$, at the price of considering that an object satisfying $p, q$ and $q^{\prime}$ would still be better (even if such an object does not exist).

In this section, one wants to be able to express that for instance, an object that satisfies properties $p_{1}$ and $p_{2}$ is acceptable, while satisfying $p_{1}, p_{2}$ and $p_{3}$ makes it less acceptable, or even not acceptable at all. Thus, we have four types of situations given the subset of properties it (more or less) satisfies:

- the object is acceptable (to some degree) because it satisfies all the properties in a subset $S$, and it would be less acceptable (or not acceptable at all) in case one of the properties in $S$ is not satisfied at all, or maybe less satisfied;

- the object is acceptable (to some degree) because it satisfies a subset $S$ of properties included in a larger subset $T$ of satisfied properties, and it would be still as much acceptable in case some particular property in $T-S$ is not satisfied at all, or is less satisfied: i.e., there is no positive synergy between properties in 
$S$ and properties in $T-S$, or in other words, one is indifferent about satisfying properties in $T-S$, whence all properties in $S$ are satisfied;

- the object is rejected, not acceptable (to some degree) because it satisfies all the properties in a subset $R$, and it would be less rejected (or not rejected at all) in case one of the properties in $R$ is not satisfied at all, or maybe less satisfied;

- the object is rejected, because it satisfies all the properties in a subset $U$ that includes a forbidden combination of properties $R$, the property in $U-R$ having no influence on this state of fact.

In the following, for coping with the problem of the representation of positive and negative properties, we propose to use a pair of basic possibility assignments $\left(\sigma_{*}, \sigma^{*}\right)$ where $\sigma_{*}(S)$ describes to what extent an object satisfying all properties in $S$ is satisfactory, while $\sigma^{*}(S)$ expresses to what extent an object satisfying all properties in $S$ is rejected. A consistency condition, as in any bipolar representation of this kind [7], should hold, namely

$$
\forall S \sigma_{*}(S) \leq 1-\sigma^{*}(S)
$$

in order to express that an acceptable objects are included among those that are not rejected.

\section{B. A pair of Sugeno integrals for negative synergies}

It is assumed that the information is in a conjunctive form, and that there are two types of important sets of properties involved, playing positive and negative roles. Acceptance of objects are defined with respect to them. To model this situation we need to have two basic possibilistic assignments: $\sigma_{*}$ and $\sigma^{*}$. We first examine the binary case.

- Let $\mathbf{P}^{+}$the familly of the sets of properties playing positive role. We define $\sigma_{*}$ by $\sigma_{*}(T)=1$ if $T \in \mathbf{P}^{+}$, $\sigma_{*}(T)=0$ if $T \notin \mathbf{P}^{+}$and $\sigma_{*}(\emptyset)=0$. According to these sets of properties an object $x$ is acceptable if there exists $T \in \mathbf{P}^{+}$such that $x$ fully satisfies all the properties belonging to $T$. So we have $\oint_{\mu_{*}}(x)=1$ where $\mu_{*}$ is the fuzzy measure associated to $\sigma_{*}$.

- Let $\mathbf{P}^{-}$the family of the sets of properties playing negative role. We define $\sigma^{*}$ by $\sigma^{*}(T)=1$ if $T \in \mathbf{P}^{-}$, $\sigma^{*}(T)=0$ if $T \notin \mathbf{P}^{-}$and $\sigma^{*}(\emptyset)=0$. According to these sets of properties an object $x$ is acceptable if $x$ doesn't satisfy all the properties of each subset. So we have $\oint_{\mu^{*}}(x)=0$ where $\mu^{*}$ is the fuzzy measure associated to $\sigma^{*}$.

So $x$ is an acceptable object iff $\oint_{\mu_{*}}(x)=1$ and $\oint_{\mu^{*}}(x)=0$.

In the example presented in the previous section, the $\sigma$ used is $\sigma_{*}$. We need to consider that each subset of properties different from $\{p, q\}$ and $\{p, r\}$ play a negative role. Consequently we define $\sigma^{*}$ such that $\sigma^{*}(\emptyset)=0$, $\sigma^{*}(\{p, q\})=0, \sigma^{*}(\{p, r\})=0$ and 1 everywhere. Hence we have $\oint_{\mu^{*}}(1,1,1)=1 \neq 0$, which entails that $(1,1,1)$ is no longer acceptable.
Example 2.4 Let us go back to the example presented for gradual properties with $[0,1]$-valued measures. $\mathcal{P}=$ $\left\{p_{1}, p_{2}, p_{3}, p_{4}\right\}$ and acceptable objects are those satisfying $p_{1}$ and $p_{2}$ with $\sigma_{*}\left(\left\{p_{1}\right\}\right)=1$ and $\sigma_{*}\left(\left\{p_{2}\right\}\right)=0.5$. We add that acceptable objects don't have properties $p_{3}$ and $p_{4}$ with $\sigma^{*}\left(\left\{p_{3}\right\}\right)=1 \sigma^{*}\left(\left\{p_{4}\right\}\right)=0.5$ and 0 otherwise. As presented previously according to $\oint_{\mu_{*}}, x_{2}$ and $x_{3}$ are accepted. With $\sigma^{*}$ we obtain

$\oint_{\mu^{*}}\left(x^{1}\right)=\oint_{\mu^{*}}(0.5,1,1,1)=1$,

$\oint_{\mu^{*}}\left(x^{2}\right)=\oint_{\mu^{*}}(1,0.5,1,0)=1$,

$\oint_{\mu^{*}}\left(x^{3}\right)=\oint_{\mu^{*}}(1,1,0,0)=0$,

$\oint_{\mu^{*}}\left(x^{4}\right)=\oint_{\mu^{*}}(0,1,0.4,0.2)=0.4$.

So finally only $x^{3}$ is found to be acceptable.

If we decide to be soft, $x^{4}$ would be accepted from $\oint_{\mu^{*}}$ point of view. In this case $p_{3}$ and $p_{4}$ are satisfied. But the most important property is satisfied only at level 0.4 . According to $\oint_{\mu_{*}} x^{1}$ and $x^{4}$ would be acceptable. So in this case $x 4$ could be found to be acceptable.

More generally, it can be checked that the pair of Sugeno integrals associated with the positive assignment $\sigma_{*}$ and the negative assignment $\sigma^{*}$, enables us to acknowledge the objects that are acceptable (to a degree), and those that are rejected (to a degree).

\section{SOME LINES FOR FURTHER RESEARCH}

Standard formal concept analysis only considers concepts described in intention by conjunctions of properties, while pattern structures [9] that allow any logical description [8] are more general. Moreover, it has been recently established that a possibilistic logic base gives birth to a pattern structure [1], while other set operators recently proposed for enlarging the formal concept analysis [2] can be generalized as well. This raises the question of knowing if Sugeno integrals are also pattern structures from a formal analysis point of view. This may seem quite likely if we also consider the fact that the elicitation of Sugeno integrals-based representations gives birth to a version space-like approach [14], while there exists a connection between pattern structures and version space-based conceptual learning [13]. This is an open question however. In that perspective, it may be worth mentioning that the following entailment-like relation exists between Sugeno integrals

Proposition $2 \oint_{\mu} \leq \oint_{\mu^{\prime}}$ iff $\forall S \subseteq \mathcal{P}, \exists T \subseteq S$ such that $\sigma(S) \leq \sigma^{\prime}(T)$

which somewhat parallels the entailment relation between basic probability assigments [5].

Proof If $\oint_{\mu} \leq \oint_{\mu^{\prime}}$ then for all $S \subseteq \mathcal{P}, \mu(S) \leq \mu^{\prime}(S)$ i.e $\vee_{T \subseteq S} \sigma(T) \leq \vee_{T \subseteq S} \sigma^{\prime}(T)$. For all $S \subseteq \mathcal{P}, \sigma(S) \leq$ $\vee_{T \subseteq S} \bar{\sigma}^{\prime}(T)$ which entails $\exists T \subseteq S$ such that $\sigma(S) \leq \sigma^{\prime}(T)$. If $\forall S \subseteq \mathcal{P}, \exists T \subseteq S$ such that $\sigma(S) \leq \sigma^{\prime}(T)$ then for all $S$, $\sigma(S) \leq \vee_{T \subseteq S} \sigma^{\prime}(T)$. If we choose $S_{0}$ we have $\forall S \subseteq S_{0}$, $\sigma(S) \leq \vee_{T \subseteq S_{0}} \sigma^{\prime}(T)$ so $\vee_{T \subseteq S_{0}} \sigma(T) \leq \vee_{T \subseteq S_{0}} \sigma^{\prime}(T)$ i.e $\mu_{S_{0}} \leq \mu_{S_{0}}^{\prime}$. This can be done for all $S_{0} \subseteq \mathcal{P}$ which entails $\mu \leq \mu^{\prime}$ and $\oint_{\mu} \leq \oint_{\mu^{\prime}}$. 
Moreover, the preorder between basic possibility assignments defined in the above proposition is such that

Proposition 3 If $\oint_{\mu}=\oint_{\mu^{\prime}}$ then

- $\forall p_{i} \in \mathcal{P}, \sigma\left(\left\{p_{i}\right\}\right)=\sigma^{\prime}\left(\left\{p_{i}\right\}\right)$;

- $\sigma$ and $\sigma^{\prime}$ describe the same important set of properties minimal according to inclusion: $T$ is a set of properties such that $\sigma(T)=1$ and $\forall S \subseteq T, \sigma(S) \neq 1$ if and only if $\sigma^{\prime}(T)=1$ and $\forall S \subseteq T, \sigma^{\prime}(S) \neq 1$.

Proof If $\oint_{\mu}=\oint_{\mu^{\prime}}$ then $\vee_{T \subseteq S} \sigma(T)=\vee_{T \subseteq S} \sigma^{\prime}(T)$. Considering a property $p_{i}$ one obtains $\sigma\left(\left\{p_{i}\right\}\right)=\sigma^{\prime}\left(\left\{p_{i}\right\}\right)$. Let $T$ be a set of properties such that $\sigma(T)=1$ and if $T_{1} \subset T$ then $\sigma\left(T_{1}\right)<1$. According to the previous equality, $\exists S \subseteq T$ such that $\sigma(T) \leq \sigma^{\prime}(S)$. So $\sigma^{\prime}(S)=1$ which entails $\exists S_{1} \subseteq S$ such that $\sigma^{\prime}(S) \leq \sigma\left(S_{1}\right)$. So we have $\sigma\left(S_{1}\right)=1$ with $S_{1} \subseteq T$ which is possible only if $S=$ $T$. Precisely, if there exists $T_{1} \subset T$ such that $\sigma^{\prime}\left(T_{1}\right)=1$ then the equality permits us to find a set $T_{2} \subset T$ such that $\sigma\left(T_{2}\right)=1$ which is not possible because $T$ is minimal according to the inclusion operator.

So we have $\sigma^{\prime}(T)=1$ and $\forall S \subset T \sigma^{\prime}(S)<1$. Using the equality the result is the same, beginning with $\sigma^{\prime}$ instead of $\sigma$ which concludes the proof.

The exact role played by this preorder is still to be investigated.

Other lines of research include the case where $\vee_{T \subseteq \mathcal{P}} \sigma(T)=1$ is not satisfied, then the expression (1) cannot be used. However, using the classical definition seems to imply similar results considering that an important set is a set containing a subset $S$ such that $\sigma(S)$ is the maximal value reached by $\sigma$.

Another issue is the complexity of the representation, since $\sigma$ is defined on a power set. Still, it could be defined only on some elements (using then some kind of extension principle to specify the distribution on other subsets, when their value is non zero).

Lastly, the idea of using a pair of Sugeno integrals may be found somewhat reminiscent of bi-capacities [11]; a possible link is to be further explored.

\section{CONCLUSION}

Starting in this paper from the facts that Sugeno integrals have the ability to encode Boolean formulas (without negation) on the one hand, and can deal with gradual properties on the other hand, we have made some first steps towards the use of Sugeno integrals as a representation tool, and not only as an evaluation tool, making a parallel with possibilistic logic. While possibilistic logic can represent negative synergies between properties, Sugeno integrals cannot, since they are increasing with the satisfaction degrees of the properties. The use of pairs of Sugeno integrals has been proposed for coping with this problem.

\section{REFERENCES}

[1] Z. Assaghir, M. Kaytoue, H. Prade. A possibility theoryoriented discussion of conceptual pattern structures. Proc. 4th Inter. Conf. on Scalable Uncertainty Management (SUM'10), (A. Deshpande, A. Hunter, eds.), Toulouse, Sept. 27-29, Springer, LNAI 6379, 70-83, 2010.

[2] D. Dubois, F. Dupin de Saint Cyr, H. Prade. A possibiltytheoretic view of formal concept analysis. Fundamenta Informaticae, (1-4):195-213, 2007.

[3] D. Dubois, J.-L. Marichal, H. Prade, M. Roubens, R. Sabbadin. The use of the discrete Sugeno integral in decision-making: A survey. Inter. J. of Uncertainty, Fuzziness and Knowledgebased Systems, 9, 539-561, 2001.

[4] D. Dubois, H. Prade. Evidence measures based on fuzzy information, Automatica, 21, 547-562, 1985.

[5] D. Dubois, H. Prade. A set-theoretic view of belief functions. Logical operations and approximations by fuzzy sets. Int. J. General Systems, 12, 193-226, 1986.

[6] D. Dubois, H. Prade. Possibilistic logic: a retrospective and prospective view. Fuzzy Sets and Systems, 144, 3-23, 2004.

[7] D. Dubois, H. Prade, P. Smets. Not impossible vs.guaranted possible in fusion and revision. Proc. 6th.Europ. Conf. on Symbolic and Quantitative Approach to Reasoning, Toulouse, Sept. 19-21, Springer, LNAI 2143, 522-531, 2001.

[8] S. Ferré, O. Ridoux. Introduction to logical information systems. Inf. Process. Management, 40(3):383-419, 2004.

[9] B. Ganter, S. O. Kuznetsov. Pattern structures and their pro jections. Proc. of the 9th International Conference on Conceptual Structures (ICCS'01), 129-142. Springer, 2001.

[10] B. Ganter, R. Wille. Formal Concept Analysis. Springer, 1999

[11] M. Grabisch, C. Labreuche: Bi-capacities - Part I: definition, Möbius transform and interaction. CoRR abs/0711.2114, 2007.

[12] M. Grabisch, C. Labreuche, A decade of application of the Choquet and Sugeno integrals in multi-criteria decision aid. 4OR, 6: 1-44, 2008.

[13] S. O. Kuznetsov. Learning of simple conceptual graphs from positive and negative examples. In J. M. Zytkow and J. Rauch, editors, Proc. 3rd Europ. Conf. on Principles and Practice of Knowledge Discovery in Databases, PKDD 99, Prague, Sept. 15-18, LNCS 1704, 384-391, Springer, 1999.

[14] H. Prade, A. Rico, M. Serrurier. Elicitation of Sugeno integrals: A version space learning perspective, Proc. 18th Inter. Symp. on Methodologies of Intelligent Systems (ISMIS 2009), Prague, Sept. 14-17, 2009, (J. Rauch, Z. W. Ras, P. Berka, T. Elomaa, eds.), Springer, LNAI 5722, 392-401, 2009.

[15] J.-L. Marichal. Aggregation Operations for Multicriteria Decision Aid. Ph.D.Thesis, University of Liège, Belgium, 1998.

[16] M. Sugeno. Theory of fuzzy integrals and its applications, Ph.D. Thesis, Tokyo Institute of Technology, Tokyo, 1974.

[17] M. Sugeno. Fuzzy measures and fuzzy integrals: a survey. In: Fuzzy Automata and Decision Processes, (M.M. Gupta, G.N. Saridis, and B.R. Gaines, eds.), North-Holland, 89-102, 1977. 